\title{
The Hall technique of fame?
}

Use of the Hall technique by specialist paediatric dentists: a global perspective. Br Dent J 2020; 228: 33-38.

https://doi.org/10.1038/s41415-019-1100-2.

Preformed metal crowns (PMC) have a higher success rate and longevity when compared to plastic restorations for treating class 1 or class 2 asymptomatic carious primary molars. The child-friendly and non-invasive Hall Technique (HT) was introduced in the UK in 2006 as an alternative method for treating primary molars. By simply choosing a correct sized PMC and cementing it to the appropriate carious tooth with GIC, it starves the bacteria from sugary substrates, arresting the carious lesion. Although it has recently been described as the 'gold standard' within the UK due to its high success rates, is it internationally recognised? The aim of this research article is to assess global paediatric dentists' awareness, knowledge and use of the HT in practice.

A mixture of opinions of its use was portrayed according to the surface involved and whether the tooth was cavitated or not. Sixty-eight percent stated that they would never/rarely use HT in non-cavitated class 1 lesions and $78 \%$ reported being inclined to use HT with cavitated class 2 lesions which aligned with current guidelines. Sixty-two percent accepted the use of pre-HT radiographs with ๑) $60 \%$ believing it should be within one month

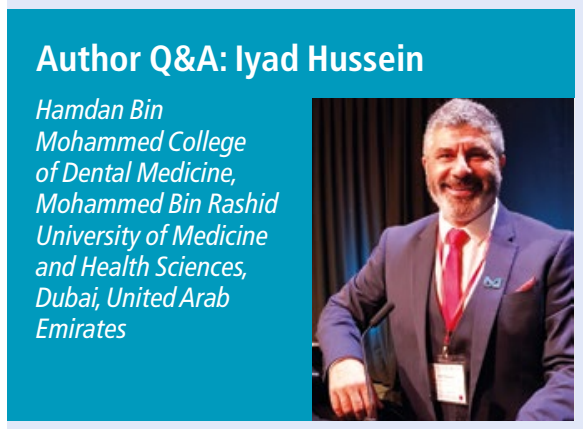

Why did you decide to undertake this study?

To plug a gap in Hall Technique related research. The HT, a high-quality evidencebased breakthrough in paediatric dentistry was introduced to UK GDPs in 2006 by the $B D J$. Despite the science; it also represented a new philosophy causing a $B D J$ war of words in the UK between those who were supportive (https://www.nature.com/ articles/sj.bdj.2008.701) and those against

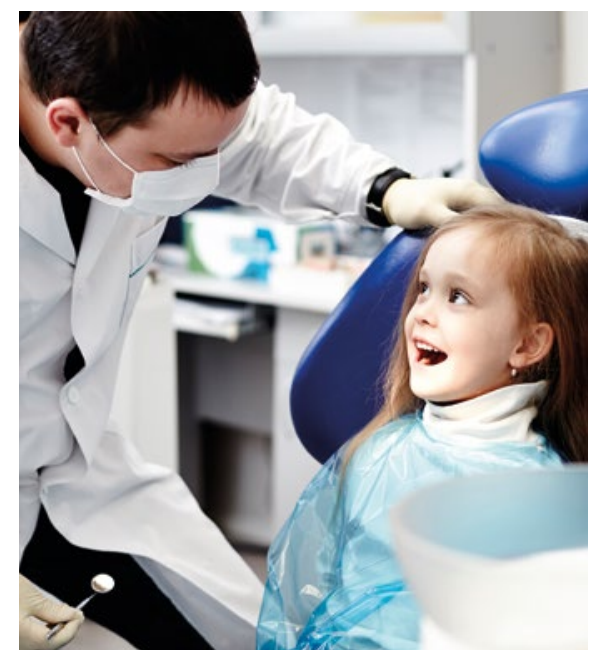

setting would be most appropriate to place a HTPMC, the highest response was for a specialist practice setting (62\%) as opposed to a GDP setting (33\%) or at undergraduate training (28\%).

Despite the $92 \%$ or higher success rate of using HT, the results portray a significant lack of confidence for HT in the paediatric dental community. This was portrayed with more than half reporting insufficient evidence supporting the use of HT and only $17 \%$ using HT as the gold standard for non-pulpal asymptomatic carious primary molars.

Even within the second decade of its use, there is still significant opposition to the HT with only $50.6 \%$ using HT from the $92 \%$ of participants that had heard of it. What was even more surprising was that several opposing comments were made mainly by academics and senior teachers within paediatric dentistry. The evidence continuously favours the use of HT, however, the need to breakdown certain knowledge barriers in managing carious lesions is still needed within global paediatric dentistry.

Sonal Gandhi

Dental Core Trainee, Northwick Park Hospital (https://www.nature.com/articles/4813693).

Fast-forward to 2018, and almost every British paediatric dentist is using the HT. Case settled? Well, yes in the UK, but not the rest of the globe - especially followers of the American Academy of Paediatric Dentistry (AAPD). The HT is not endorsed by AAPD guidelines. The global HT debate was very intense indeed, with eminent USA dentists leading the opposition to the HT, considering it serious malpractice (https:// www.aegisdentalnetwork.com/id/2017/02/ heal-or-conceal). As a British paediatric dentist university teacher who moved to Dubai, UAE (a vibrant microcosm of the world), I was exposed to supporters and opponents of the HT on the global scene. With the help of 16 colleagues, many of them AAPD diplomates (who use the HT), this research emerged to confirm the existence of a HT schism (50:50) in the world stage of paediatric dentistry.

\section{Did any of the results surprise you?}

Yes, that many emotive opposing comments came from senior academics/teachers who took part in the survey. I was also surprised about the high proportion of USA dentists who were using HT, going against the AAPD guidelines.

What do you think the next steps should be considering your findings?

The HT has been labelled the 'gold standard' in managing non-pulpal carious primary molars but also a 'a gross distortion of medicine and science. The next step, in my view, should be joint scientific meetings between the AAPD, BDA, BSPD to debate the contemporary available scientific evidence to reach a consensus, or a formal disagreement because both sides of the debate want the best for children's oral health. 